\title{
Rat Protein
}

National Cancer Institute

\section{Source}

National Cancer Institute. Rat Protein. NCI Thesaurus. Code C26534.

Proteins found in any species or strain of rat. 